\title{
Brighter Red Fluorescent Proteins by Rational Design of Triple-Decker Motif
}

\author{
Antonia T. Pandelieva, ${ }^{1}$ Miranda J. Baran, ${ }^{1}$ Guido F. Calderini, ${ }^{1}$ Jenna L. McCann, ${ }^{1}$ \\ Véronique Tremblay, ${ }^{2}$ Sabina Sarvan, ${ }^{2}$ James A. Davey, ${ }^{1}$ Jean-François Couture ${ }^{2,3, *}$ \& \\ Roberto A. Chica ${ }^{1,3, *}$ \\ ${ }^{1}$ Department of Chemistry and Biomolecular Sciences, University of Ottawa, 10 Marie-Curie, \\ Ottawa, Ontario, K1N 6N5, Canada \\ ${ }^{2}$ Ottawa Institute of Systems Biology, Department of Biochemistry, Microbiology and Immunology, \\ University of Ottawa, 451 Smyth Road, Ottawa, Ontario, K1H 8M5, Canada \\ ${ }^{3}$ Centre for Catalysis Research and Innovation, University of Ottawa, 30 Marie-Curie, Ottawa, \\ Ontario, K1N 6N5, Canada \\ ${ }^{*}$ Corresponding authors: \\ Roberto A. Chica: rchica@uottawa.ca \\ Jean-François Couture: Jean-Francois.Couture@uottawa.ca
}

Supplementary material included:

\section{Supplementary Methods}

Supplementary Figure 1. mRojoA mutants containing aromatic residues at positions 63 and 143 do not form mature chromophore.

Supplementary Figure 2. pH profiles.

Supplementary Table 1. pKa values for various RFPs.

Supplementary References 


\section{Supplementary Methods}

Materials. All reagents used were of the highest available purity. Restriction enzymes and DNAmodifying enzymes were from New England Biolabs. Synthetic oligonucleotides were obtained from Integrated DNA Technologies and Eurofins MWG Operon. Ni-NTA agarose resin was obtained from Promega and lysozyme was purchased from AMRESCO. All aqueous solutions were prepared using water purified with a Barnstead Nanopure Diamond system.

Mutagenesis. The $m$ RojoA gene cloned into the pET11a plasmid (Novagen) via Ndel/BamHI ${ }^{1}$ served as a template for mutagenesis by overlap extension ${ }^{2}$ using Vent DNA polymerase (New England Biolabs). Briefly, external primers were used in combination with sets of complementary pairs of degenerate oligonucleotides containing the desired mutations in individual PCR reactions. The resulting overlapping fragments were gel-purified (Omega Bio-Tek) and recombined by overlap extension PCR. The resulting amplicons were digested with Ndel and BamHI, gel-purified, and ligated into pET11a expression vector with T4 ligase (New England Biolabs). All constructs were verified by sequencing the entire reading frame.

Screening. The DNA libraries prepared as described above were transformed into chemically competent E. coli BL21-Gold (DE3) cells (Agilent). Colonies were picked into individual wells of V96 MicroWell polypropylene plates (Nunc) containing $200 \mu \mathrm{L}$ of Luria-Bertani Broth supplemented with $100 \mu \mathrm{g} / \mathrm{mL}$ ampicillin and $10 \%$ glycerol. The plates were covered with a sterile, breathable rayon membrane (VWR) and incubated overnight at $37^{\circ} \mathrm{C}$ with shaking. After incubation, these mother plates were used to inoculate sterile V96 MicroWell polypropylene plates ("daughter" plates) containing $300 \mu \mathrm{L}$ of Overnight Express Instant TB medium (Novagen) supplemented with ampicillin per well. Daughter plates were sealed with breathable membranes and incubated overnight $\left(37^{\circ} \mathrm{C}, 250 \mathrm{rpm}\right)$. After incubation, cells were harvested by centrifugation 
$\left(3000 \times \mathrm{g}, 30\right.$ minutes, $\left.4^{\circ} \mathrm{C}\right)$ and cell pellets were washed twice with phosphate buffered saline $\mathrm{pH} 7.4$ (PBS). Washed cell pellets were then incubated at $4{ }^{\circ} \mathrm{C}$ for $72 \mathrm{~h}$ to allow chromophore maturation. These pellets were resuspended in PBS and transferred to a Fluotrac 96-well plate (Greiner Bio-One) for screening. Screening was performed using a Tecan Infinite M1000 plate reader equipped with a plate stacker. Emission $\left(\lambda_{\mathrm{ex}}=565 \mathrm{~nm}\right)$ and excitation spectra $\left(\lambda_{\mathrm{em}}=650\right.$ $\mathrm{nm}$ ) were measured.

Protein Expression and Purification. Proteins were expressed in 500-mL cultures (LuriaBertani Broth supplemented with $100 \mu \mathrm{g} \mathrm{mL}-1$ ampicillin) of E. coli BL21-Gold (DE3) cells transformed with pET11a containing the mutated genes. Cultures were grown at $37^{\circ} \mathrm{C}$ with shaking until an optical density of 0.6 at $600 \mathrm{~nm}$ was reached. At this point, protein expression was induced with $1 \mathrm{mM}$ isopropyl $\beta$-D-1-thiogalactopyranoside, and cells were incubated overnight at $16{ }^{\circ} \mathrm{C}$ with shaking. Following incubation, cells were harvested by centrifugation and lysed with an EmulsiFlex-B15 cell disruptor (Avestin). Proteins were purified by immobilized metal affinity chromatography according to the manufacturer's protocol. The eluted fractions were desalted by gel filtration using EconoPAC 10DG columns (Bio-Rad) into PBS. 


\section{Supplementary Figures and Tables}
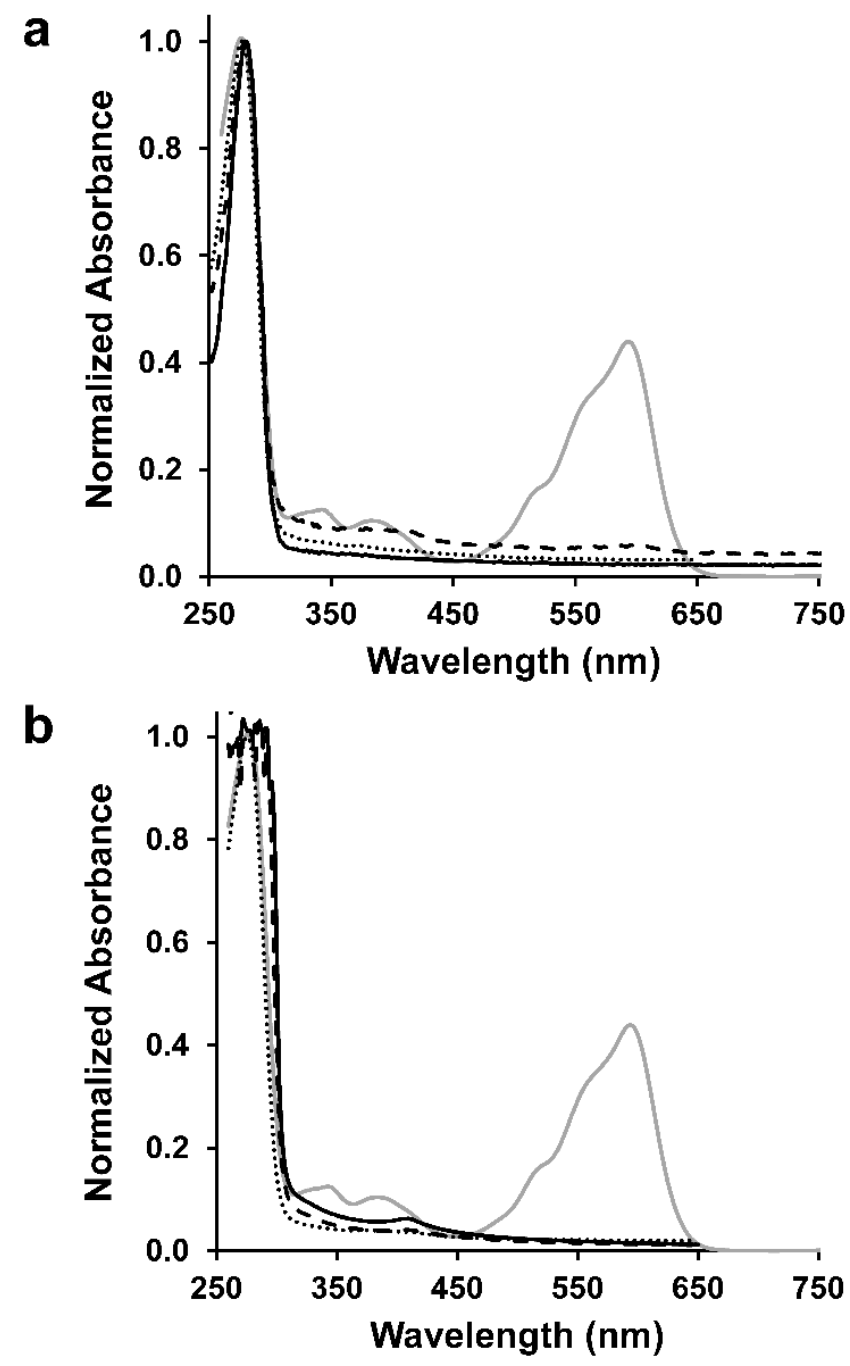

Supplementary Figure 1. mRojoA mutants containing aromatic residues at positions 63 and 143 do not form mature chromophore. (a) Absorption spectra of mRojoA single mutants $\mathrm{P} 63 \mathrm{H}, \mathrm{P} 63 \mathrm{~F}$, and P63Y are shown as dashed black, dotted black, and solid black lines, respectively. Absorption spectrum of $m R$ ojoA is shown as solid grey line. (b) Absorption spectra of mRojo-VYWL, mRojo-TYWV, and mRojo-VYWV are shown as dashed black, dotted black, and solid black lines, respectively. Absorption spectrum of $m$ RojoA is shown as solid grey line. All spectra are normalized to the $280 \mathrm{~nm}$ peak. 

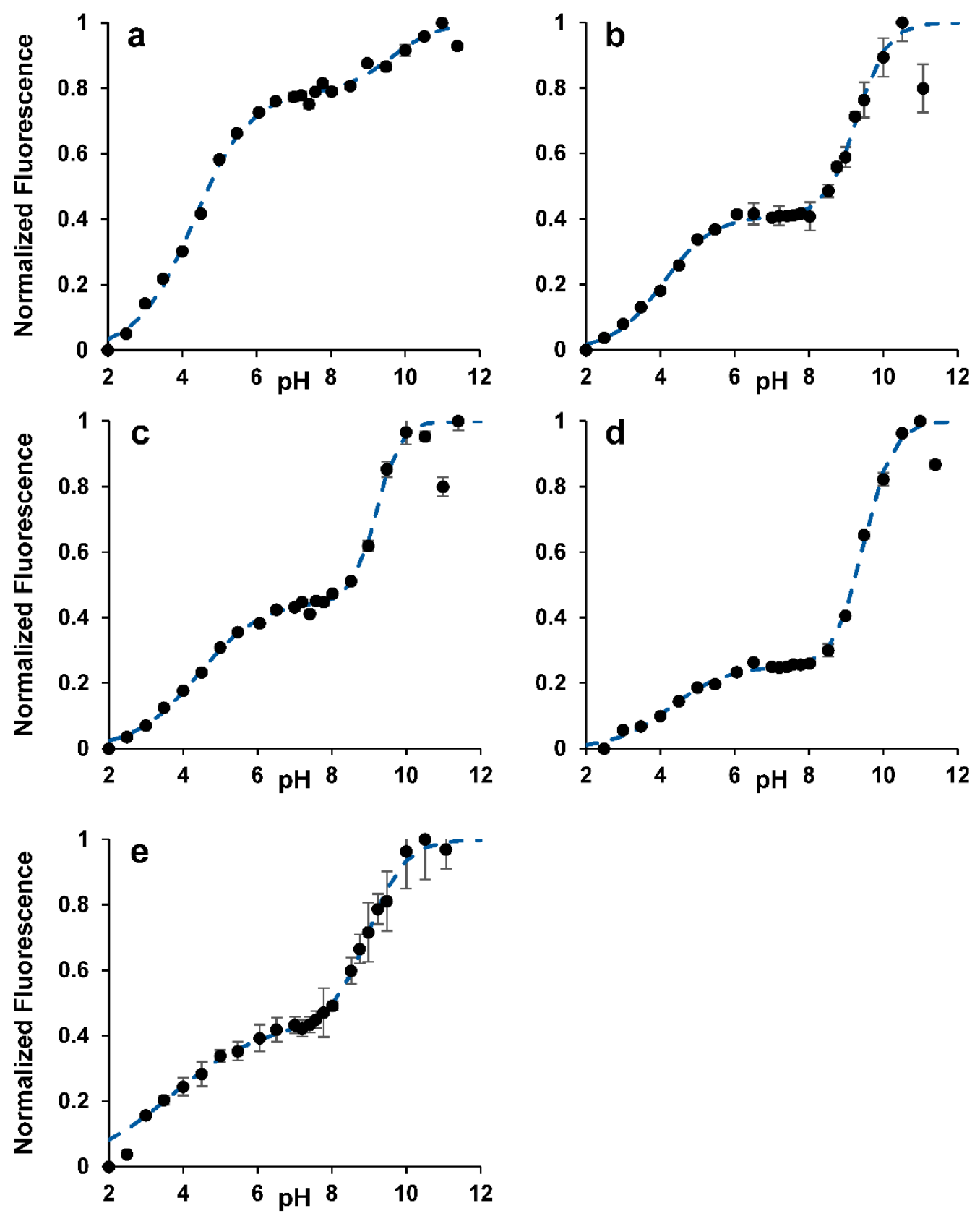

Supplementary Figure 2. pH profiles. (a) mRojoA, (b) mRojo-VYGV, (c) mRojo-VFAV, (d) mRojo-VHSV, and (e) mRojo-THSL. 
Supplementary Table 1. pKa values for various RFPs.

\begin{tabular}{lcccc}
\hline \multicolumn{1}{c}{ Protein } & $\mathrm{pKa}_{1}$ & $\mathrm{n}_{1}{ }^{a}$ & $\mathrm{pKa}_{2}$ & $\mathrm{n}_{2}{ }^{a}$ \\
\hline mRojoA & 4.6 & 0.6 & 9.6 & 0.7 \\
mRojo-THSL & 3.7 & 0.4 & 9.0 & 0.9 \\
mRojo-THSV & 3.2 & 0.7 & 9.3 & 0.9 \\
mRojo-VHSV & 4.3 & 0.6 & 9.5 & 1.1 \\
mRojo-TFAL & 6.1 & 0.4 & 9.9 & 1.8 \\
mRojo-TFAV & 4.3 & 0.5 & 9.3 & 1.1 \\
mRojo-VFAV & 4.4 & 0.5 & 9.2 & 1.4 \\
mRojo-TYGL & 4.5 & 0.5 & 8.9 & 1.1 \\
mRojo-TYGV & 3.9 & 0.6 & 9.3 & 0.8 \\
mRojo-VYGV & 4.3 & 0.7 & 9.1 & 1.1 \\
mRojo-VYGL & 4.1 & 0.5 & 9.1 & 1.0 \\
mRojo-VPGV & 4.2 & 0.6 & 9.5 & 1.0 \\
\hline
\end{tabular}

${ }^{a}$ Hill coefficients obtained by least squares fitting.

\section{Supplementary References}

1. Chica, R. A., Moore, M. M., Allen, B. D., and Mayo, S. L. (2010) Generation of longer emission wavelength red fluorescent proteins using computationally designed libraries, Proc Natl Acad Sci U S A 107, 20257-20262.

2. Ho, S. N., Hunt, H. D., Horton, R. M., Pullen, J. K., and Pease, L. R. (1989) Site-directed mutagenesis by overlap extension using the polymerase chain reaction, Gene $77,51-59$. 